\title{
PLD3 epigenetic changes in the hippocampus of Alzheimer's disease
}

\author{
Idoia Blanco-Luquin ${ }^{1}$, Miren Altuna ${ }^{1,2}$, Javier Sánchez-Ruiz de Gordoa ${ }^{1,2}$, Amaya Urdánoz-Casado', Miren Roldán', \\ María Cámara ${ }^{2}$, Victoria Zelaya ${ }^{3}$, María Elena Erro ${ }^{1,2}$, Carmen Echavarri ${ }^{1,4}$ and Maite Mendioroz ${ }^{1,2^{*}}$
}

\begin{abstract}
Background: Whole-exome sequencing has revealed a rare missense variant in PLD3 gene (rs145999145) to be associated with late onset Alzheimer's disease (AD). Nevertheless, the association remains controversial and little is known about the role of PLD3 in AD. Interestingly, PLD3 encodes a phospholipase that may be involved in amyloid precursor protein (APP) processing. Our aim was to gain insight into the epigenetic mechanisms regulating PLD3 gene expression in the human hippocampus affected by AD.

Results: We assessed PLD3 mRNA expression by qPCR and protein levels by Western blot in frozen hippocampal samples from a cohort of neuropathologically confirmed pure AD cases and controls. Next, we profiled DNA methylation at cytosine-phosphate-guanine dinucleotide $(\mathrm{CpG})$ site resolution by pyrosequencing and further validated results by bisulfite cloning sequencing in two promoter regions of the PLD3 gene. A 1.67-fold decrease in PLD3 mRNA levels ( $p$ value $<0.001$ ) was observed in the hippocampus of AD cases compared to controls, and a slight decrease was also found by Western blot at protein level. Moreover, PLD3 mRNA levels inversely correlated with the average area of $\beta$-amyloid burden (tau-b $=-0,331 ; p$ value $<0.01$ ) in the hippocampus. A differentially methylated region was identified within the alternative promoter of PLD3 gene showing higher DNA methylation levels in the AD hippocampus compared to controls ( $21.7 \pm 4.7 \%$ vs. $18.3 \pm 4.8 \% ; p$ value $<0.05$ ).
\end{abstract}

Conclusions: PLD3 gene is downregulated in the human hippocampus in AD cases compared to controls. Altered epigenetic mechanisms, such as differential DNA methylation within an alternative promoter of PLD3 gene, may be involved in the pathological processes of AD. Moreover, PLD3 mRNA expression inversely correlates with hippocampal $\beta$-amyloid burden, which adds evidence to the hypothesis that PLD3 protein may contribute to AD development by modifying APP processing.

Keywords: PLD3, Alzheimer's disease, Epigenetics, DNA methylation, Gene and protein expression, Hippocampus, APP, Lysosome

\section{Background}

Alzheimer's disease (AD) is a genetically complex process where $\varepsilon 4$ allele of the $A P O E$ gene (APOE4) is by far the best-established genetic susceptibility risk factor. In addition, genome-wide association studies have revealed a considerable number of small-effect common variants in genes related to $\mathrm{AD}[1-3]$. However, those

\footnotetext{
* Correspondence: maitemendilab@gmail.com

${ }^{1}$ Neuroepigenetics Laboratory-Navarrabiomed, Complejo Hospitalario de Navarra, Universidad Pública de Navarra (UPNA), IdiSNA (Navarra Institute for Health Research), C/ Irunlarrea, 3, 31008 Pamplona, Navarra, Spain

${ }^{2}$ Department of Neurology, Complejo Hospitalario de Navarra- IdiSNA (Navarra Institute for Health Research), C/ Irunlarrea, 3, 31008 Pamplona, Navarra, Spain

Full list of author information is available at the end of the article
}

variants do not explain the full heritability of this disease. More recently, novel sequencing technologies are enabling the identification of other rare genetic variants that could potentially contribute to the development of sporadic AD. Notable recent discoveries in this area include rare disease variants in TREM2, UNC5C, AKAP9, TM2D3, ADAM10, and PLD3 genes [2, 4, 5].

PLD3 (phospholipase D family, member 3) (OMIM * 615698) gene is located at chromosome 19q13.2 and encodes a lysosomal protein that belongs to the phospholipase D (PLD) superfamily, which catalyzes the hydrolysis of membrane phospholipids. However, PLD3 catalytic function has not yet been demonstrated $[6,7]$. PLD3 gene is highly expressed in the brain of healthy

(c) The Author(s). 2018 Open Access This article is distributed under the terms of the Creative Commons Attribution 4.0 International License (http://creativecommons.org/licenses/by/4.0/), which permits unrestricted use, distribution, and 
controls, particularly in several brain regions vulnerable to AD pathology, such as frontal, temporal, and occipital cortices and hippocampus, but reduced in neurons from $\mathrm{AD}$ brains [3, 8]. Nevertheless, little is known on the regulation, the function, and the involvement of PLD3 in $\mathrm{AD}$ pathogenesis.

Interestingly, controversial association exists about this gene conferring increased risk for the development of AD. Cruchaga et al. performed whole-exome sequencing on $\mathrm{AD}$ patients and identified a rare missense variant (rs145999145) in exon 7 of the PLD3 gene which resulted in a val232-to-met (V232M) substitution [8]. Their results revealed that carriers of the PLD3 coding variant had a twofold increased risk for late onset $\mathrm{AD}$. Moreover, they showed that PLD3 influences amyloid precursor protein (APP) processing, acting as a negative regulator, since PLD3 overexpression in cultured neuroblastoma cells correlated with lower intracellular APP, extracellular $A \beta 42$, and $A \beta 40$ levels and that PLD3 protein could be co-immunoprecipitated with APP. In that regard, Satoh et al. showed an accumulation of PLD3 on neuritic plaques in $\mathrm{AD}$ brains and suggested a key role for PLD3 in the pathological processes of AD [9].

Other authors confirmed that PLD3 gene variant $\mathrm{V} 232 \mathrm{M}$ was associated with $\mathrm{AD}$ risk and significantly lower cognitive function [10] providing a systematic view of the involvement of PLD3 in $\mathrm{AD}$ at genetic, mRNA, and protein level expression. However, additional studies were not able to define an essential role of PLD3 rare variants in AD [11], neither to support an important contribution of PLD3 rare variants in the etiology of $\mathrm{AD}$, given the high variability of the frequency of PLD3 Val232Met variant across populations [12]. Indeed, follow-up studies have questioned the role of PLD3 rare variants in $\mathrm{AD}$, obtaining negative replication data [13-15] and suggesting a more complex role of PLD3 in the etiology of the disease.

Keeping in mind the results mentioned above, we wanted to gain insight into the epigenetic mechanisms regulating PLD3 expression in order to add evidence to the potential contribution of PLD3 to AD. Further knowledge on these mechanisms may provide opportunities for new AD therapeutic strategies. Here, we profiled PLD3 gene expression and methylation in the human hippocampus, one of the most vulnerable brain regions to AD. To that end, we selected a cohort of neuropathologically defined "pure" AD cases and controls to measure hippocampal PLD3 expression by quantitative PCR and Western blot. Next, we explored the correlation of PLD3 expression with AD neuropathological changes. Finally, DNA methylation levels at two distinct promoter regions of the PLD3 gene were assessed by pyrosequencing and bisulfite cloning sequencing.

\section{Methods}

\section{Human hippocampal samples and neuropathological examination}

Brain hippocampal samples from $30 \mathrm{AD}$ patients and 12 controls were provided by Navarrabiomed Brain Bank. After death, half brain specimens from donors were cryopreserved at $-80{ }^{\circ} \mathrm{C}$. Neuropathological examination was completed following the usual recommendations [16] and according to the updated National Institute on Aging-Alzheimer's Association guidelines [17]. Assessment of $\beta$-amyloid deposition was carried out by immunohistochemical staining of paraffin-embedded sections $(3-5 \mu \mathrm{m}$ thick) with a mouse monoclonal (S6F/3D) anti $\beta$-amyloid antibody (Leica Biosystems Newcastle Ltd., Newcastle upon Tyne, UK). Evaluation of neurofibrillary pathology was performed with a mouse monoclonal antibody anti-human PHF-TAU, clone AT-8, (Tau AT8) (Innogenetics, Gent, Belgium), which identifies hyperphosphorylated tau (p-tau) [18]. The reaction product was visualized using an automated slide immunostainer (Leica Bond Max) with Bond Polymer Refine Detection (Leica Biosystems, Newcastle Ltd.).

To avoid spurious findings related to multiprotein deposits, "pure" AD cases with deposits of only p-tau and $\beta$-amyloid were eligible for the study and controls were free of any pathological protein aggregate. This approach maximizes chances of finding true associations with $\mathrm{AD}$, even though reducing the final sample size. A summary of characteristics of subjects included in this study is shown in Additional file 1: Table S1. AD subjects were older than controls $(82.3 \pm 11.3$ versus $50.7 \pm 21.5 ; p$ value $<0.01)$, and no differences were found regarding gender ( $p$ value $=0.087)$. The postmortem interval $(\mathrm{PMI})$ were not significantly different between groups $(8.2 \pm 4.4 \mathrm{~h}$ in the control group versus $7.9 \pm 7.1 \mathrm{~h}$ in the $\mathrm{AD}$ group; $p$ value $=0.91$ ).

\section{PLD3 mRNA expression analysis by RT-qPCR}

Total RNA was isolated from hippocampal homogenates using RNeasy Lipid Tissue Mini kit (QIAGEN, Redwood City, CA, USA), following the manufacturer's instructions. Genomic DNA was removed with recombinant DNase (TURBO DNA-free ${ }^{\mathrm{Tm}}$ Kit, Ambion, Inc., Austin, TX, USA). RNA integrity was checked by $1.25 \%$ agarose gel electrophoresis under denaturing conditions. Concentration and purity of RNA were both evaluated with NanoDrop spectrophotometer. Only RNA samples showing a minimum quality index $(260 \mathrm{~nm} / 280 \mathrm{~nm}$ absorbance ratios between 1.8 and 2.2 and $260 \mathrm{~nm} / 230 \mathrm{~nm}$ absorbance ratios higher than 1.8) were included in the study. Complementary DNA (cDNA) was reverse transcribed from 1500 ng total RNA with SuperScript ${ }^{\circ}$ III First-Strand Synthesis Reverse Transcriptase (Invitrogen, Carlsbad, CA, USA) after priming with oligo-d $(\mathrm{T})$ and random primers. RT-qPCR reactions were performed in 
triplicate with Power SYBR Green PCR Master Mix (Invitrogen, Carlsbad, CA, USA) in a QuantStudio 12K Flex Real-Time PCR System (Applied Biosystems, Foster City, CA, USA) and repeated twice within independent cDNA sets. Sequences of primer pair were designed using Real Time PCR tool (IDT, Coralville, IA, USA) and are listed in Additional file 1: Table S2. Relative expression level of PLD3 mRNA in a particular sample was calculated as previously described [19] and $A C T B$ gene was used as the reference gene to normalize expression values.

\section{PLD3 protein expression analysis by Western blot}

Human hippocampus tissue from patients and control samples was lysed with $100 \mu \mathrm{L}$ lysis buffer containing urea, thiourea, and DTT. After centrifugation at $35.000 \mathrm{rpm}$ for $1 \mathrm{~h}$ at $15^{\circ} \mathrm{C}$, extracted proteins were quantified following the Bradford-Protein Assay (Bio-Rad, Hercules, CA, USA) by using a spectrophotometer.

Next, $5 \mu \mathrm{g}$ of protein per sample were resolved in $4-20 \%$ Criterion TGX stain-free gels (Bio-Rad) and electrophoretically transferred onto nitrocellulose membranes using a Trans-blot Turbo transfer system (25 V, $7 \mathrm{~min}$ ) (Bio-Rad). Equal loading of the gel was assessed by stain-free digitalization and by Ponceau staining. Membranes were probed with rabbit anti-human PLD3 primary antibody (Sigma-Aldrich; 1:250) in 5\% nonfat milk and incubated with peroxidase-conjugated anti-rabbit secondary antibody (Cell Signaling; 1:2000). Immunoblots were then visualized by exposure to an enhanced chemiluminescence Clarity Western ECL Substrate (Bio-Rad) using a ChemidocMP Imaging System (Bio-Rad). Expression levels of PLD3 were standardized by the corresponding band intensity of GAPDH (Calbiochem; 1:10000).

\section{Quantitative assessment of $\beta$-amyloid and $p$-tau deposits in hippocampal samples}

In order to quantitatively assess the $\beta$-amyloid and $p$-tau burden for further statistical analysis, we applied a method to quantify protein deposits, as described in detail elsewhere [20]. In brief, hippocampal sections were examined after performing immunostaining with anti $\beta$-amyloid and anti $\mathrm{p}$-tau antibodies. Focal deposit of $\beta$-amyloid, including neuritic, immature, and compact plaques [21], was analyzed with the ImageJ software. Moreover, $\beta$-amyloid plaque count, referred to as amyloid plaque score (APS), was measured. Finally, p-tau deposit was also analyzed with ImageJ software in order to obtain an average quantitative measure of the global p-tau deposit for each section.

\section{PLD3 methylation measurement by pyrosequencing}

Genomic DNA was isolated from frozen hippocampal tissue by phenol-chloroform method [22]. Next, $500 \mathrm{ng}$ of genomic DNA was bisulfite converted using the
EpiTect Bisulfite Kit (Qiagen, Redwood City, CA, USA) according to the manufacturer's protocol. Primers to amplify and sequence two promoter regions of PLD3 were designed with PyroMark Assay Design version 2.0.1.15 (Qiagen) (Additional file 1: Table S2), and PCR reactions were carried out on a VeritiTM Thermal Cycler (Applied Biosystems, Foster City, CA, USA). Next, $20 \mu \mathrm{l}$ of biotinylated PCR product was immobilized using streptavidin-coated sepharose beads (GE Healthcare Life Sciences, Piscataway, NJ, USA) and $0.3 \mu \mathrm{M}$ sequencing primer was annealed to purified DNA strands. Pyrosequencing was performed using the PyroMark Gold Q96 reagents (Qiagen) on a PyroMark $^{\mathrm{m}}$ Q96 ID System (Qiagen). For each particular cytosine-phosphate-guanine dinucleotide $(\mathrm{CpG})$, methylation levels were expressed as percentage of methylated cytosines over the sum of total cytosines. Unmethylated and methylated DNA samples (EpiTect PCR Control DNA Set, Qiagen) were used as controls for the pyrosequencing reaction.

\section{PLD3 methylation validations by bisulfite cloning sequencing}

Bisulfite-converted genomic DNA was used to validate pyrosequencing results. Primer pair sequences were designed by MethPrimer [23] and are listed in Additional file 1: Table S2. PCR products were cloned using the TopoTA Cloning System (Invitrogen, Carlsbad, CA, USA), and a minimum of 10-12 independent clones were sequenced for each examined subject and region. Methylation graphs were obtained with QUMA software [24].

\section{Statistical data analysis}

Statistical analysis was performed with SPSS 21.0 (IBM, Inc., USA). Before performing differential analysis, we checked that all continuous variables showed a normal distribution, as per one-sample Kolgomorov-Smirnov test and the normal quantil-quantil (QQ) plots. Data represents the mean \pm standard deviation (SD). Significance level was set at $p$ value $<0.05$. Statistical significance for PLD3 mRNA levels and pyrosequecing intergroup differences was assessed by $T$ test. One-way analysis of variance (ANOVA) followed by Games-Howell post hoc analysis was used to analyze differences in the expression levels of PLD3 mRNA between Braak and Braak stage groups. A logistic regression model (ENTER method) was fit to assess the independent association of PLD3 mRNA levels with $\mathrm{AD}$ status, using gender and age as covariates. Kendall's tau-b correlation coefficient was used to determine correlation between AD-related pathology and PLD3 mRNA expression levels. Difference between two bisulfite cloning sequencing groups was evaluated with Mann-Whitney $U$ test. GraphPad Prism version 6.00 for Windows (GraphPad Software, La Jolla, CA, USA) 
was used to draw graphs except for methylation figures that were obtained by QUMA software.

\section{Results}

PLD3 expression is downregulated in Alzheimer's disease hippocampus

As the first step in this study, we measured PLD3 mRNA expression levels by real-time quantitative PCR (RT-qPCR) in the hippocampus of AD patients compared to controls. Five samples did not pass the RNA quality threshold (see the "Methods" section) and so were not included in the experiments. Eventually, $26 \mathrm{AD}$ cases were compared to 11 controls. As shown in Fig. 1a, PLD3 mRNA levels were significantly decreased by 1.67 -fold in the hippocampus of $\mathrm{AD}$ cases compared to controls $[p$ value $<0.001]$. Next, a disease-staging analysis was performed to investigate changes of PLD3 mRNA levels depending on the AD severity measured by Braak \& Braak staging [21]. We found that PLD3 mRNA levels were significantly reduced across Braak \& Braak stages [ $p$ value $<0.005$; Fig. 1b]. Games-Howell post hoc analysis revealed that PLD3 mRNA expression was significantly different between control and Braak stages III-IV [ $p$ value $<0.05]$ and between control and Braak stages V-VI $[p$ value $<0.05]$ (Fig. 1).
Then, to identify adjusted estimates of the association of PLD3 mRNA levels with $\mathrm{AD}$ status (control $=0$; $\mathrm{AD}$ $=1$ ), a logistic regression model was designed. Age and gender were included into the model to adjust for potentially confounding variables. As shown in Table 1, PLD3 mRNA expression levels remained as an independent predictor of $\mathrm{AD}$ status after adjusting for age and gender $[p$ value $<0.05]$ (Table 1 ).

In order to examine whether the decrease in PLD3 mRNA levels in the AD hippocampus extended to the protein level, a Western blot analysis was performed. Protein extracts from frozen hippocampal samples that were included in the qPCR experiment were obtained, and a polyclonal antibody against a recombinant protein epitope signature tag (PrEST) of PLD3 was used. GAPDH protein detection was used as housekeeping. In line with the PLD3 mRNA expression results, we observed that PLD3 protein expression tends to be decreased in samples from hippocampus of AD patients as compared to controls (Fig. 1c).

\section{Correlation of PLD3 mRNA expression levels with p-tau and amyloid deposits}

Next, we aimed to correlate PLD3 mRNA levels with AD-related neuropathological changes in hippocampal

\section{A}

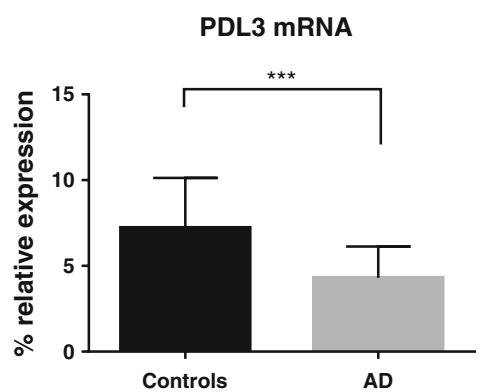

C

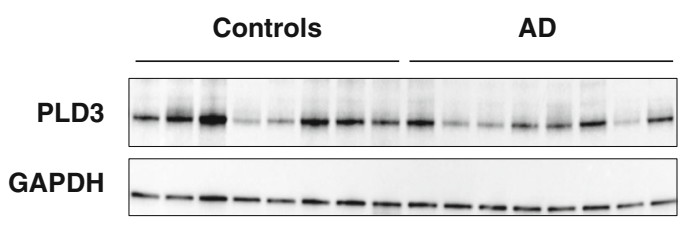

B
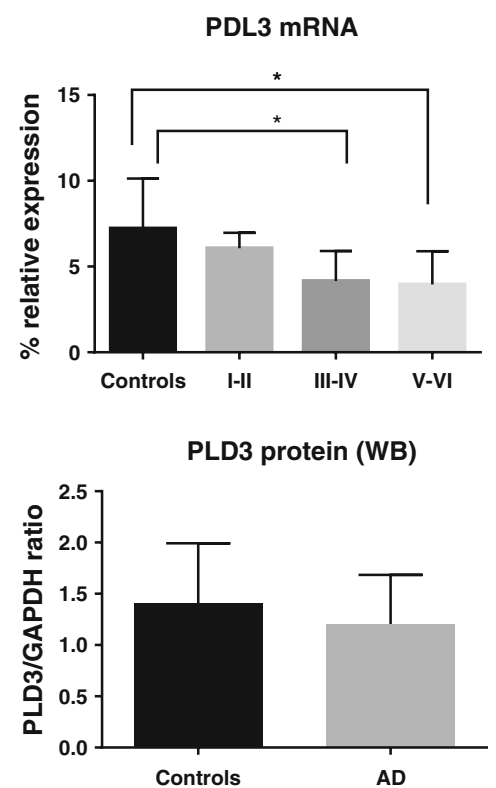

Fig. 1 PLD3 expression is decreased in human hippocampus in Alzheimer's disease (AD). a The graph shows a significant 1.67-fold decrease in PLD3 mRNA levels in AD hippocampal samples compared to control hippocampal samples. b PLD3 mRNA expression decreased across AD stages, as shown when PLD3 mRNA expression levels are sorted by Braak and Braak stages. Bars represent percentage of PLD3 mRNA expression relative to ACTB housekeeping gene expression. Vertical lines represent the standard error of the mean. ${ }^{*} p$ value $<0.05$; ${ }^{* * *} p$ value $<0.001$. c Western blot analysis of PLD3 shows a mild protein expression decrease in AD. Human hippocampus samples from controls or AD patients were loaded as labeled on top of lanes. GADPH expression is shown as reference control. The bar chart represents the quantitative measurement of the PLD3 protein relative to GAPDH protein expression 
Table 1 Adjusted logistic regression model to predict AD status

\begin{tabular}{lllll}
\hline Variable & $\mathrm{B}$ & Wald & $p$ value & OR \\
\hline PLD3 mRNA levels & -0.544 & 4.212 & $0.040^{*}$ & 0.581 \\
Gender (female) & 0.613 & 0.286 & 0.593 & 1.847 \\
Age $<65$ years old & 2.774 & 5.981 & $0.014^{*}$ & 16.02 \\
Constant & -1.494 & 0.254 & 0.614 & 0.224
\end{tabular}

Alzheimer status (control $=0 ; A D=1$ ) was considered as the dependent variable and PLD3 mRNA expression levels, gender, and age were included as covariates

$B$ regression coefficient, $O R$ odds ratio

${ }^{*} p$ value $<0.05$

sections. In brief, $\beta$-amyloid and hyperphosphorylated tau (p-tau) burden were measured and averaged for each subject by a semi-automated quantitative method by using the ImageJ software (see the "Methods" section). The amyloid plaque score (APS) was also recorded. As $\beta$-amyloid and p-tau data were not normally distributed, the non-parametric Kendall's tau-b correlation coefficient was used. We found that the average area of $\beta$-amyloid burden in the hippocampus was inversely correlated with PLD3 mRNA levels [tau- $\mathrm{b}=-0,331 ; p$ value $<0.01$ ], and accordingly, an inverse association was found between APS and PLD3 mRNA levels [tau-b $=-0,319 ; p$ value $<0.01$ ] Regarding p-tau deposits, a statistically significant correlation was found for an inverse correlation [tau-b $=-0,306$; $p$ value $<0.01]$.

DNA methylation in PLD3 is increased in hippocampus of AD cases compared to controls

DNA methylation levels of regulatory regions in the genome modulate the expression of related or nearby genes. Thus, we tested whether DNA methylation levels in PLD3 gene were also altered in the AD hippocampus. PLD3 gene is located in the long arm of chromosome 19 (19q13.2) and has two distinct CpG island-containing promoter regions as shown by the UCSC Genome Browser website [25] (Fig. 2a). The principal promoter, which is placed at the $5^{\prime}$ end of the gene, contains a 553 bp CpG island (chr19:40854181-40854733; GRCh37/ hg19) while an alternative promoter overlapping exon 2 contains a smaller 207 bp CpG island (chr19:40871 618-40871824; GRCh37/hg19). Pyrosequencing primers were designed to amplify and sequence specific CpGs within both promoters regions (P_prom CpG1 and CpG2 for the principal promoter and A_prom CpG1 and CpG2 for the alternative promoter) (Fig. 2a).

We observed that the principal promoter of PLD3 was mostly demethylated [mean $\pm \mathrm{SD}, 1.8 \pm 2.9 \%$ ], as it corresponds to the constitutive promoter of an actively expressed gene. Average DNA methylation levels were slightly higher in AD cases compared to controls only for P_prom CpG2 [2.6 $\pm 3.13 \%$ vs. $0 \pm 0 \% ; p$ value $<0.001]$. The alternative promoter showed intermediate levels of
DNA methylation [20.5 $\pm 4.91 \%]$. A_prom CpG1 showed a statistical trend to be highly methylated in AD cases compared to controls $[23 \pm 7.8 \%$ vs. $18.4 \pm$ $6.2 \% ; p$ value $=0.09]$ and a statistically significant difference in DNA methylation levels was observed for A_prom CpG2 in AD cases compared to controls [21.5 $\pm 5.8 \%$ vs. $15.3 \pm 3.8 \% ; p$ value $<0.01]$.

Next, we sought to replicate pyrosequencing results by extending the initial cohort with additional AD and control hippocampal samples for which DNA was available. These samples came from Navarrabiomed Brain Bank and were used to increase the sample size for the methylation experiments. Eventually, $36 \mathrm{AD}$ patients and 18 controls were analyzed by pyrosequencing. In the principal promoter, average DNA methylation levels showed a trend to be higher in $\mathrm{AD}$ cases compared to controls at P_prom CpG2 [2.3 $\pm 2 \%$ vs. $1 \pm 2.4 \% p$ value $=0.094]$. In the alternative promoter, no differences were found for A_prom CpG1 between AD cases and controls [ $p$ value $>0.05]$. However, we observed a statistically significant difference in DNA methylation levels for A_prom CpG2 between $\mathrm{AD}$ cases and controls $[21.7 \pm 4.7 \%$ vs. $18.3 \pm$ $4.8 \% ; p$ value $<0.05$ ], pointing to a differentially methylated region located within the alternative promoter of PLD3 in the AD hippocampus (Fig. 2b). In order to test whether A_prom CpG2 methylation was an independent predictor of $\mathrm{AD}$ status (control $=0 ; \mathrm{AD}=1$ ), a binary logistic regression model was performed. After adjusting for age and gender, A_prom CpG2 methylation levels remain as an independent predictor of AD (Additional file 1: Table S3).

We validated the pyrosequencing results and extended the methylation local mapping by using bisulfite cloning sequencing in two independent amplicons overlapping both PLD3 promoter regions. DNA methylation percentage was measured at $\mathrm{CpG}$ site resolution and further averaged across all the $\mathrm{CpG}$ sites for each amplicon. In line with the previous pyrosequencing results, we found that average DNA methylation levels of the amplicon at PLD3 principal promoter were very low and showed no differences between AD patients and controls (Fig. 2c). On the contrary, average DNA methylation levels of the amplicon at PLD3 alternative promoter were increased in $\mathrm{AD}$ patients compared to controls $[19.1 \pm 7.8 \%$ vs. $6 \pm$ $4 \%$; $p$ value $<0.05$ ] (Fig. 2c).

Since DNA methylation is one of the major mechanisms to regulate gene expression, we analyzed the correlation between PLD3 mRNA expression and PLD3 DNA methylation in our sample set. No significant correlation was found between expression and DNA methylation measured by pyrosequencing [A_prom CpG1 $r=-0.264, p$ value $=0.114 ;$ A_prom CpG2 $r=-0.275, p$ value $=0.110]$. However, a significant inverse correlation was observed between expression and DNA methylation 


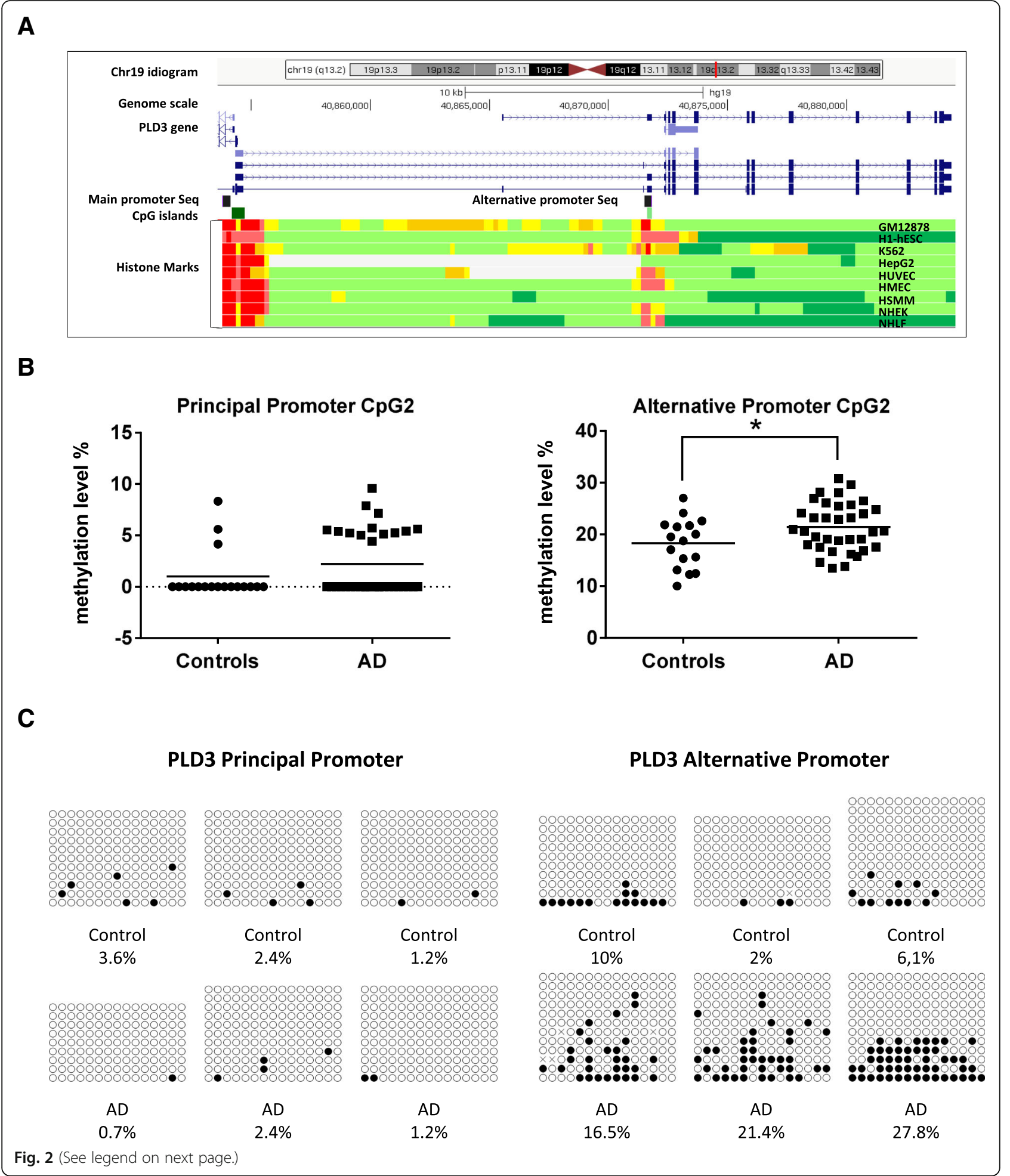




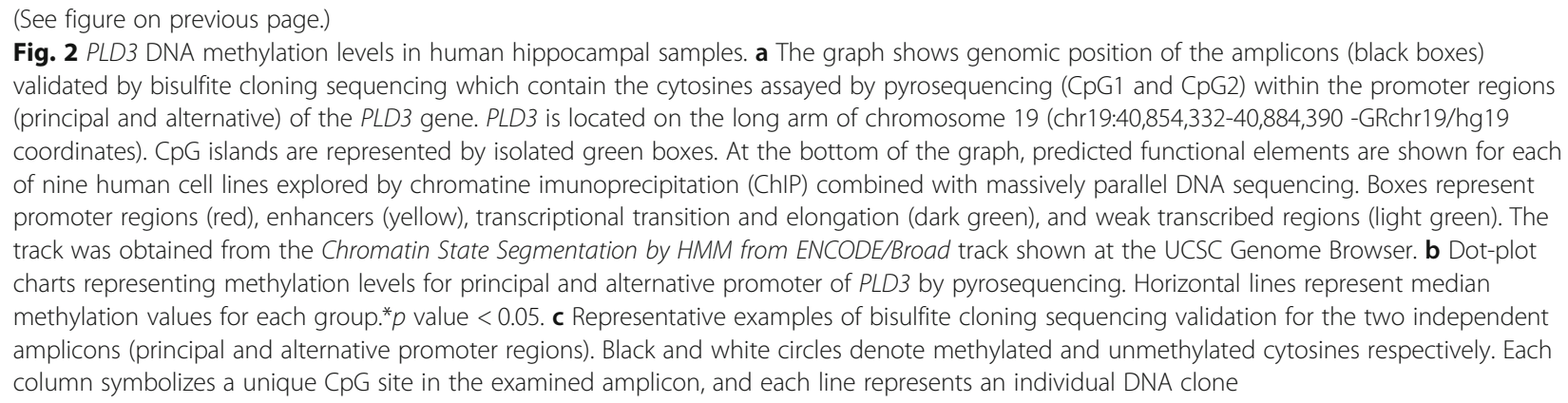

in the PLD3 alternative promoter measured by bisulfite cloning sequencing $[r=-0.683 ; p$ value $<0.05]$.

\section{Discussion}

We report PLD3 gene to be downregulated at both transcript and protein level in the human hippocampus affected by AD. In addition, we show that the decrease in PLD3 mRNA expression inversely correlates with $\beta$-amyloid burden in the hippocampus. An important finding of this study is that an alternative promoter of PLD3 gene is differentially methylated in the hippocampus of $\mathrm{AD}$ patients compared to controls suggesting that epigenetic disturbances in PLD3 may occur in the pathological process of AD.

Our results showing a reduction in PLD3 expression in the AD hippocampus add to previous evidence supporting the idea that PLD3 gene is downregulated in brain areas affected by AD processes [8, 9]. Cruchaga et al. [8] used data from genome-wide transcriptomics in laser-captured neurons from $33 \mathrm{AD}$ cases and 16 controls (GEO dataset GSE5281) [26] to reveal that PLD3 gene expression was significantly lower in $\mathrm{AD}$ cases compared to controls. In addition, Satoh et al. found a marginal reduction in PLD3 mRNA levels in the frontal cortex of $7 \mathrm{AD}$ cases compared to 14 non-AD subjects, including other neurodegenerative disorders such as amyotrophic lateral sclerosis and Parkinson disease [9]. In agreement with the previous results, we observed a statistically significant decrease in PLD3 mRNA expression in the hippocampus, a vulnerable region to $\mathrm{AD}$ pathology, and also show that PLD3 is reduced across Braak \& Braak stages indicating that PLD3 is somehow related to the progressive neurodegenerative processes of AD.

A number of different mechanisms could explain the decrease in PLD3 gene expression in the AD hippocampus, including the progressive loss of neuronal populations, changes in cellular composition with increasing astrogliosis in late stages of $\mathrm{AD}$, or cell-type-specific decrease in PLD3 gene expression. A limitation of the present study is that it has been designed on a tissue-specific basis, and therefore, changes in gene expression at cell-specific level, including neuron-specific level, cannot be assessed. In fact, the ratio of cellular components in the human hippocampus may change across different stages of $\mathrm{AD}$. In this case, and if the expression of PLD3 were cell type specific, the gene expression changes observed globally in the hippocampus could be attributed to the loss of a given cell population and not reflect actual PLD3 expression changes. However, the fact of having found epigenetic modifications in the same sample set would support the existence of a true alteration in the regulation of PLD3 gene expression. To know whether the difference in PLD3 gene expression is driven by a decreased expression in neurons or by changes in the ratio of cell populations in the brain of $\mathrm{AD}$ patients, other technologies, such as the emerging single-cell techniques, should be used.

The reduction in PLD3 expression is in line with the classical $\beta$-amyloid cascade hypothesis of $\mathrm{AD}$, since PLD3 protein seems to act as a negative regulator of APP processing [8]. It has been shown that knockdown of PLD3 expression in cells results in higher levels of extracellular $A \beta 42$ and $A \beta 40$ levels, and conversely, overexpression of PLD3 is associated with reduced extracellular A $\beta 42$ and A $\beta 40$ levels [8]. Furthermore, PLD3 protein is accumulated in neuritic plaques in human $A D$ brains [9]. Indeed, it has been demonstrated that PLD3 protein can be co-immunoprecipitated with APP in cultured cells [8]. Even more, PLD3 protein has been recently characterized as a novel endosome-to-Golgi retrieval gene that regulates the endosomal protein sorting, whose loss of function results in increased processing of APP [27]. Accordingly, we have found an inverse correlation between PLD3 mRNA expression levels and the burden of hippocampal $\beta$-amyloid assessed by two measurements, averaged deposit of $\beta$-amyloid and amyloid plaque score (APS). All these data supports the notion that PLD3 protein could display a protective effect against AD pathology through its role in APP trafficking, as other authors have previously suggested [27].

Interestingly, PLD3 protein is co-expressed with other lysosomal proteins [9], including progranulin, which regulates lysosomal functioning and is also accumulated in neuritic plaques $[9,28]$. Moreover, PLD3 protein is 
required to preserve the structure of lysosomes in vivo and, therefore, impairment of the endosomal-lysosomal systems has been proposed as an alternative mechanism by which PLD3 could contribute to the development of AD [29]. Most interestingly, another genetic variant in PLD3, p.Leu308Pro, was recently found to cause autosomal dominant spinocerebellar ataxia [30], a neurodegenerative condition where lysosomal disturbances are thought to be crucial $[31,32]$. As an additional alternative explanation, PLD3 might also influence AD pathological processes by altering adult neurogenesis since PLD3 gene expression seems to be turned on at late stages of neurogenesis [33].

Finally, we describe an altered pattern of DNA methylation within an alternative promoter of the PLD3 gene in the human hippocampus affected by AD. To our knowledge, no previous reports on altered DNA methylation in PLD3 gene have been published and very little is known about regulation of PLD3 gene expression. The alternative promoter of PLD3 is placed $\sim 17,500$ bp downstream the principal promoter overlapping exon 2. It contains a small CpG island and is conserved across several cell types (Fig. 2a). In our study, it was found to be differentially methylated showing higher DNA methylation levels in $\mathrm{AD}$ patients than in controls. Since DNA methylation of $\mathrm{CpG}$ islands is one of the major epigenetic mechanisms that influence gene expression, our results indicate that altered DNA methylation at this particular regulatory region might contribute to downregulate PLD3 expression in AD.

In this regard, we also show a significant correlation between PLD3 mRNA expression and DNA methylation in our dataset when measured by bisulfite cloning sequencing, while the pyrosequencing results did not show correlation with expression. It is intriguing why the significant correlation is found only for the bisulfite cloning sequencing results. First of all, although not significant, an inverse correlation appears in the statistical analysis for the pyrosequencing results. However, it is only a statistical trend. One possible explanation would be that DNA methylation levels measured by bisulfite cloning sequencing average the methylation levels of an extended genomic region ( $15 \mathrm{CpGs})$, and therefore, this result may be more close to the real functional effect of methylation on gene expression than the result of individual CpGs.

Epigenetic disturbances are increasingly being described for a number of genes related to AD, including genes harboring rare variants that contribute to developing AD [34-40]. In this sense, our work provides new knowledge about the epigenetic alterations involved in gene transcription regulation in key brain regions for the development of AD. Additionally, our results support the involvement of PLD3 in the pathology of AD.

\section{Conclusions}

To sum up, this study confirms that PLD3 gene is downregulated in the hippocampus of AD patients. Moreover, PLD3 expression inversely correlates with $\beta$-amyloid burden, which adds evidence to the hypothesis that PLD3 protein may contribute to $\mathrm{AD}$ development through modifying APP processing. Having identified a differentially methylated region in an alternative promoter of PLD3, our study suggests that epigenetic disturbances in PLD3 gene may be involved in the pathological processes of $\mathrm{AD}$.

\section{Additional file}

Additional file 1: Table S1. Brain sample set analyzed in our study. Table S2. RT-qPCR and bisulfite PCR primers. Table S3. Adjusted logistic regression model to predict $A D$ status. (PDF $465 \mathrm{~kb}$ )

\section{Abbreviations}

AD: Alzheimer's disease; ANOVA: Analysis of variance; APOE4: $\varepsilon 4$ allele of the APOE gene; APP: Amyloid precursor protein; APS: Amyloid plaque score; CDNA: Complementary DNA; CpG: Cytosine-phosphate-guanine dinucleotide; GEO: Gene Expression Omnibus; PLD3: Phospholipase D family, member 3; PrEST: Protein epitope signature tag; p-tau: Hyperphosphorylated tau; QQ: Quantil-quantil plots; RT-qPCR: Real-time quantitative PCR; Tau AT8: Mouse monoclonal antibody anti-human PHF-TAU, clone AT-8; UCSC: University of California, Santa Cruz; V232M: Val232-to-met substitution

\section{Acknowledgements}

We want to kindly thank Teresa Tuñón M.D., Ph.D (Department of Pathology, Complejo Hospitalario de Navarra, technical support), Federico García-Bragado M.D., Ph.D (Department of Pathology, Complejo Hospitalario de Navarra, technical support), Iván Méndez M.D., (Department of Internal Medicine, Hospital García Orcoyen, technical editing), and Isabel Gil M.D. (Navarrabiomed BrainBank, technical support) for their help. Finally, we are very grateful to the patients and relatives that generously donor the brain tissue to the Navarrabiomed Brain Bank.

\section{Funding}

This work was supported by the Spanish Government through grants from the Institute of Health Carlos III (FIS PI13/02730 \& PI17/02218), jointly funded by the European Regional Development Fund (ERDF), European Union, "A way of shaping Europe"; the Regional Basque Government through a grant from The Basque Foundation for Health Innovation and Research (BIOEF) (BIO12/ALZ/ 007), a grant from Fundación Caja-Navarra; and the Trans-Pyrenean Biomedical Research Network (REFBIO). In addition, AUC received a grant "Doctorados industriales 2018-2020" founded by the Government of Navarra and MM received a grant "Programa de intensificación" funded by Fundación Bancaria "la Caixa" and Fundación Caja-Navarra.

\section{Availability of data and materials}

The datasets generated and analyzed during the current study are available from the corresponding author on reasonable request.

\section{Authors' contributions}

IBL contributed to the acquisition of the data, analysis and interpretation of the data, and drafting/revising of the manuscript for content. MR contributed to the acquisition of the data and drafting/revising of the manuscript for content. JSR contributed to the analysis and interpretation of the data ( $p$-tau and amyloid deposits), sorting of the patients into different stages, and acquisition of the image data. AU contributed to the analysis of the data, figure drawing, and drafting/revising of the manuscript for content. MR contributed to the running of the experiments and was involved in the interpretation of the data. MC contributed to the acquisition of the data and drafting/revising of the manuscript for content. MVZ participated in the revision of the subject diagnosis and classification of patients and 
contributed to the drafting/revising the manuscript for content. MEE contributed to the analysis of the results and drafting/revising of the manuscript for content. CE contributed to the acquisition of the data diagnosis of the subjects, and drafting/revising of the manuscript for content. MM contributed to the drafting/revising of the manuscript for content, study concept and design, analysis and interpretation of the data, acquisition of the data, statistical analysis, study supervision, and obtaining the funding. All authors read and approved the final manuscript.

\section{Ethics approval and consent to participate}

The Ethics Committee of the "Complejo Hospitalario de Navarra" approved the use of human subjects for this study (Pyto 90/2014). Written informed consent was obtained from all subjects or next of kin.

\section{Consent for publication}

Not applicable.

\section{Competing interests}

The authors declare that they have no competing interests.

\section{Publisher's Note}

Springer Nature remains neutral with regard to jurisdictional claims in published maps and institutional affiliations.

\section{Author details}

${ }^{1}$ Neuroepigenetics Laboratory-Navarrabiomed, Complejo Hospitalario de Navarra, Universidad Pública de Navarra (UPNA), IdiSNA (Navarra Institute for Health Research), C/ Irunlarrea, 3, 31008 Pamplona, Navarra, Spain. 2Department of Neurology, Complejo Hospitalario de Navarra- IdiSNA (Navarra Institute for Health Research), C/ Irunlarrea, 3, 31008 Pamplona, Navarra, Spain. ${ }^{3}$ Department of Pathology, Complejo Hospitalario de NavarraIdiSNA (Navarra Institute for Health Research), 31008 Pamplona, Navarra, Spain. ${ }^{4}$ Hospital Psicogeriátrico Josefina Arregui, 31800 Alsasua, Navarra, Spain

Received: 23 June 2018 Accepted: 27 August 2018

\section{Published online: 12 September 2018}

\section{References}

1. Bettens K, Sleegers K, Van Broeckhoven C. Genetic insights in Alzheimer's disease. Lancet Neurol. 2013;12(1):92-104. https://doi.org/10.1016/S14744422(12)70259-4

2. Zhu JB, Tan CC, Tan L, Yu JT. State of play in Alzheimer's disease genetics. J Alzheimers Dis. 2017;58(3):631-59. https://doi.org/10.3233/JAD-170062.

3. Humphries C, Kohli MA. Rare variants and transcriptomics in Alzheimer disease. Curr Genet Med Rep. 2014;2(2):75-84. https://doi.org/10.1007/s40142-014-0035-9.

4. Giri M, Zhang M, Lü Y. Genes associated with Alzheimer's disease: an overview and current status. Clin Interv Aging. 2016;11:665-81. https://doi. org/10.2147/CIA.S105769.

5. Guimas Almeida C, Sadat Mirfakhar F, Perdigão C, Burrinha T. Impact of lateonset Alzheimer's genetic risk factors on beta-amyloid endocytic production. Cell Mol Life Sci. 2018; https://doi.org/10.1007/s00018-018-2825-9.

6. Munck A, Böhm C, Seibel NM, Hashemol Hosseini Z, Hampe W. Hu-K4 is a ubiquitously expressed type 2 transmembrane protein associated with the endoplasmic reticulum. FEBS J. 2005;272(7):1718-26. https://doi.org/10.1111/ j.1742-4658.2005.04601.x.

7. Gonzalez AC, Schweizer M, Jagdmann S, Bernreuther C, Reinheckel T, Saftig $P$, et al. Unconventional trafficking of mammalian phospholipase D3 to lysosomes. Cell Rep. 2018;22(4):1040-53. https://doi.org/10.1016/j.celrep. 2017.12.100.

8. Cruchaga C, Karch CM, Jin SC, Benitez BA, Cai Y, Guerreiro R, et al. Rare coding variants in the phospholipase D3 gene confer risk for Alzheimer's disease. Nature. 2014:505(7484):550-4. https://doi.org/10.1038/nature12825.

9. Satoh J, Kino Y, Yamamoto Y, Kawana N, Ishida T, Saito Y, et al. PLD3 is accumulated on neuritic plaques in Alzheimer's disease brains. Alzheimers Res Ther. 2014;6(9):70. https://doi.org/10.1186/s13195-014-0070-5.

10. Engelman CD, Darst BF, Bilgel M, Vasiljevic E, Koscik RL, Jedynak BM, et al. The effect of rare variants in TREM2 and PLD3 on longitudinal cognitive function in the Wisconsin Registry for Alzheimer's Prevention. Neurobiol Aging. 2018:66: 177.e1-5. https://doi.org/10.1016/j.neurobiolaging.2017.12.025.
11. Zhang DF, Fan Y, Wang D, Bi R, Zhang C, Fang Y, et al. PLD3 in Alzheimer's disease: a modest effect as revealed by updated association and expression analyses. Mol Neurobiol. 2016;53(6):4034-45. https://doi.org/10.1007/s12035015-9353-5.

12. van der Lee SJ, Holstege H, Wong TH, Jakobsdottir J, Bis JC, Chouraki V, et al. PLD3 variants in population studies. Nature. 2015;520(7545):E2-3. https:// doi.org/10.1038/nature14038

13. Cacace R, Van den Bossche T, Engelborghs S, Geerts N, Laureys A, Dillen L, et al. Rare variants in PLD3 do not affect risk for early-onset Alzheimer disease in a European Consortium Cohort. Hum Mutat. 2015;36(12):1226-35. https://doi.org/10.1002/humu.22908.

14. Lambert JC, Grenier-Boley B, Bellenguez C, Pasquier F, Campion D, Dartigues JF, et al. PLD3 and sporadic Alzheimer's disease risk. Nature. 2015:520(7545): E1. https://doi.org/10.1038/nature14036.

15. Heilmann S, Drichel D, Clarimon J, Fernández V, Lacour A, Wagner $\mathrm{H}$, et al. PLD3 in non-familial Alzheimer's disease. Nature. 2015;520(7545):E3-5. https://doi.org/10.1038/nature14039.

16. Bell JE, Alafuzoff I, Al-Sarraj S, Arzberger T, Bogdanovic N, Budka H, et al. Management of a twenty-first century brain bank: experience in the BrainNet Europe consortium. Acta Neuropathol. 2008;115(5):497-507. https://doi.org/10.1007/s00401-008-0360-8.

17. Montine TJ, Phelps CH, Beach TG, Bigio EH, Cairns NJ, Dickson DW, et al. National Institute on Aging-Alzheimer's Association guidelines for the neuropathologic assessment of Alzheimer's disease: a practical approach. Acta Neuropathol. 2012;123(1):1-11. https://doi.org/10.1007/ s00401-011-0910-3.

18. Braak H, Alafuzoff I, Arzberger T, Kretzschmar H, Del Tredici K. Staging of Alzheimer disease-associated neurofibrillary pathology using paraffin sections and immunocytochemistry. Acta Neuropathol. 2006;112(4):389-404. https://doi.org/10.1007/s00401-006-0127-z.

19. Livak KJ, Schmittgen TD. Analysis of relative gene expression data using real-time quantitative PCR and the 2(-Delta Delta C(T)) Method. Methods (San Diego, Calif). 2001;25(4):402-8. https://doi.org/10.1006/meth.2001.1262.

20. Celarain N, Sánchez-Ruiz de Gordoa J, Zelaya MV, Roldán M, Larumbe R, Pulido $L$, et al. TREM2 upregulation correlates with 5-hydroxymethycytosine enrichment in Alzheimer's disease hippocampus. Clin Epigenetics. 2016:8:37. https://doi.org/10.1186/s13148-016-0202-9.

21. Braak H, Braak E. Neuropathological stageing of Alzheimer-related changes. Acta Neuropathol. 1991;82(4):239-59.

22. Miller SA, Dykes DD, Polesky HF. A simple salting out procedure for extracting DNA from human nucleated cells. Nucleic Acids Res. 1988;16(3):1215.

23. Li LC, Dahiya R. MethPrimer: designing primers for methylation PCRs. Bioinformatics. 2002;18(11):1427-31.

24. Kumaki Y, Oda M, Okano M. QUMA: quantification tool for methylation analysis. Nucleic Acids Res. 2008;36(Web Server issue):W170-5. https://doi. org/10.1093/nar/gkn294.

25. Kent WJ, Sugnet CW, Furey TS, Roskin KM, Pringle TH, Zahler AM, et al. The human genome browser at UCSC. Genome research. 2002;12(6):996-1006. https://doi.org/10.1101/gr.229102. Article published online before print in May 2002

26. Liang WS, Reiman EM, Valla J, Dunckley T, Beach TG, Grover A, et al. Alzheimer's disease is associated with reduced expression of energy metabolism genes in posterior cingulate neurons. Proc Natl Acad Sci U S A. 2008;105(11):4441-6. https://doi.org/10.1073/pnas.0709259105.

27. Mukadam AS, Breusegem SY, Seaman MNJ. Analysis of novel endosome-toGolgi retrieval genes reveals a role for PLD3 in regulating endosomal protein sorting and amyloid precursor protein processing. Cell Mol Life Sci. 2018; https://doi.org/10.1007/s00018-018-2752-9.

28. Tanaka Y, Suzuki G, Matsuwaki T, Hosokawa M, Serrano G, Beach TG, et al. Progranulin regulates lysosomal function and biogenesis through acidification of lysosomes. Hum Mol Genet. 2017;26(5):969-88. https://doi. org/10.1093/hmg/ddx011.

29. Fazzari P, Horre K, Arranz AM, Frigerio CS, Saito T, Saido TC, et al. PLD3 gene and processing of APP. Nature. 2017;541(7638):E1-2. https://doi.org/10.1038/ nature21030.

30. Nibbeling EAR, Duarri A, Verschuuren-Bemelmans CC, Fokkens MR, Karjalainen JM, Smeets CJLM, et al. Exome sequencing and network analysis identifies shared mechanisms underlying spinocerebellar ataxia. Brain. 2017: 140(11):2860-78. https://doi.org/10.1093/brain/awx251.

31. Unno T, Wakamori M, Koike M, Uchiyama $Y$, Ishikawa $K$, Kubota $H$, et al. Development of Purkinje cell degeneration in a knockin mouse model 
reveals lysosomal involvement in the pathogenesis of SCA6. Proc Natl Acad Sci U S A. 2012;109(43):17693-8. https://doi.org/10.1073/pnas.1212786109.

32. Alves $S$, Cormier-Dequaire F, Marinello M, Marais T, Muriel MP, Beaumatin F, et al. The autophagy/lysosome pathway is impaired in SCA7 patients and SCA7 knock-in mice. Acta Neuropathol. 2014;128(5):705-22. https://doi.org/ 10.1007/s00401-014-1289-8.

33. Pedersen KM, Finsen B, Celis JE, Jensen NA. Expression of a novel murine phospholipase D homolog coincides with late neuronal development in the forebrain. J Biol Chem. 1998;273(47):31494-504.

34. Bakulski KM, Dolinoy DC, Sartor MA, Paulson HL, Konen JR, Lieberman AP, et al. Genome-wide DNA methylation differences between late-onset Alzheimer's disease and cognitively normal controls in human frontal cortex. J Alzheimers Dis. 2012;29(3):571-88. https://doi.org/10.3233/jad-2012-111223.

35. Sanchez-Mut JV, Aso E, Heyn H, Matsuda T, Bock C, Ferrer I, et al. Promoter hypermethylation of the phosphatase DUSP22 mediates PKA-dependent TAU phosphorylation and CREB activation in Alzheimer's disease. Hippocampus. 2014;24(4):363-8. https://doi.org/10.1002/hipo.22245.

36. Lunnon K, Smith R, Hannon E, De Jager PL, Srivastava G, Volta M, et al. Methylomic profiling implicates cortical deregulation of ANK1 in Alzheimer's disease. Nat Neurosci. 2014;17(9):1164-70. https://doi.org/10.1038/nn.3782.

37. De Jager PL, Srivastava G, Lunnon K, Burgess J, Schalkwyk LC, Yu L, et al. Alzheimer's disease: early alterations in brain DNA methylation at ANK1, BIN1, RHBDF2 and other loci. Nat Neurosci. 2014;17(9):1156-63. https://doi. org/10.1038/nn.3786.

38. Yu L, Chibnik LB, Srivastava GP, Pochet N, Yang J, Xu J, et al. Association of Brain DNA methylation in SORL1, ABCA7, HLA-DRB5, SLC24A4, and BIN1 with pathological diagnosis of Alzheimer disease. JAMA Neurol. 2015;72(1): 15-24. https://doi.org/10.1001/jamaneurol.2014.3049.

39. Watson CT, Roussos P, Garg P, Ho DJ, Azam N, Katsel PL, et al. Genomewide DNA methylation profiling in the superior temporal gyrus reveals epigenetic signatures associated with Alzheimer's disease. Genome Med. 2016;8(1):5. https://doi.org/10.1186/s13073-015-0258-8.

40. Celarain N, Sanchez-Ruiz de Gordoa J, Zelaya MV, Roldan M, Larumbe R, Pulido L, et al. TREM2 upregulation correlates with 5-hydroxymethycytosine enrichment in Alzheimer's disease hippocampus. Clinical Epigenetics. 2016; 8:37. https://doi.org/10.1186/s13148-016-0202-9.

Ready to submit your research? Choose BMC and benefit from:

- fast, convenient online submission

- thorough peer review by experienced researchers in your field

- rapid publication on acceptance

- support for research data, including large and complex data types

- gold Open Access which fosters wider collaboration and increased citations

- maximum visibility for your research: over $100 \mathrm{M}$ website views per year

At $\mathrm{BMC}$, research is always in progress.

Learn more biomedcentral.com/submissions 